\title{
Analytic Investigation of a Reaction-diffusion Brusselator Model with the Time-space Fractional Derivative
}

\begin{abstract}
It is well known that many models in nonlinear science are described by fractional differential equations in which an unknown function appears under the operation of a derivative of fractional order. In this study, we propose a reaction-diffusion Brusselator model from the viewpoint of the Jumarie's modified Riemann-Liouville fractional derivative. Based on the $\left(\mathrm{G}^{\prime} / \mathrm{G}\right)$-expansion method, various kinds of exact solutions are obtained. Our results could be used as a starting point for numerical procedures as well.
\end{abstract}

Keywords: fractional calculus, Brusselator model, $\left(\mathrm{G}^{\prime} / \mathrm{G}\right)$ expansion method

MSC ${ }^{\circledR}$ (2010). 26A33, 34A08, 35A20, 35K57

\footnotetext{
İsmail Aslan: Department of Mathematics, Izmir Institute of Technology, Urla, Izmir 35430, Turkey.

E-mail: ismailaslan@iyte.edu.tr
}

\section{Introduction}

The usefulness of fractional calculus to solve various problems in nonlinear science is well known. Applications can be seen in various research areas such as electro analytical chemistry, electrochemical processes, porous media, bioengineering, transport processes, robotics, fractal theory, noise simulations, etc [1-5]. Consequently, the development of fractional derivatives has been of great interest recently. Depending upon the problem under investigation, quite a number of different definitions of fractional derivatives are given in the literature. For instance, the commonly used fractional derivatives are the Riesz derivative, the Riemann-Liouville derivative, the Caputo derivative, the Sonin-Letnikov derivative, the Marchaud derivative, the Weyl derivative, the Grünwald-Letnikov derivative and others. These fractional derivatives have their advantages and disadvantages. In recent years, fractional differential equations (FDEs) have played a crucial role in various fields such as chemistry, biology, control theory, mechanics, signal processing, economics, electricity and image processing. Up to now, there have been several numerical/analytical methods proposed for solving the space and/or time FDEs. For example, the variational iteration method [6], the homotopy perturbation method [7], the Adomian decomposition method [8], differential transform method [9], finite difference method [10], finite element method [11], first integral method [12] and fractional sub-equation method [13]. However, the justmentioned methods are very restricted, so they cannot be used to tackle equations of numerous realistic scenarios.

Though several fields of fractional calculus are already well established, there is plenty of substantial work still to be done for the extension of fractional calculus to distinct subjects such as the reaction-diffusion equations. These evolutionary type equations are inherent in many scientific areas such as heat conductivity in physics, chemical diffusion processes in chemistry, kinetics of enzyme in biology and so forth. Motivated by this idea, we shall be concerned with the following reaction-diffusion Brusselator model of fractional order

$$
\begin{aligned}
& D_{t}^{\alpha} u-K D_{x}^{2 \beta} u-u^{2} v+B u=0, \\
& D_{t}^{\alpha} v-K D_{x}^{2 \beta} v+u^{2} v-B u=0,
\end{aligned}
$$

where $0<\alpha \leq 1,0<\beta \leq 1 ; D_{t}^{\alpha}$ and $D_{x}^{2 \beta}$ denote the Jumarie's modified Riemann-Liouville fractional derivatives [14] (in time) of order $\alpha$ and (in space) of order $2 \beta$, respectively; $B$ is a constant parameter and $K$ is the diffusion coefficient; the quantities $u(x, t)$ and $v(x, t)$ describe the (positive) concentrations. The classical counterpart (that is, the case $\alpha=\beta=1$ ) of the fractional Brusselator model (1) arises in the modeling of certain chemical reaction-diffusion processes. It was introduced by Prigogine and Lefever [15] as a model for an autocatalytic oscillating chemical reaction. The name comes from the hometown of the scientists who proposed it. It arises from the following four intermediate reaction steps

$$
\begin{aligned}
A & \rightarrow X, \\
B+X & \rightarrow Y+D, \\
2 X+Y & \rightarrow 3 X, \\
X & \rightarrow E,
\end{aligned}
$$


where $A$ and $B$ are input chemicals, $D$ and $E$ are output chemicals, $X$ and $Y$ are intermediates [16-18]. It is well known that the Brusselator model has been applied to various problems in both chemistry and biology.

The main purpose of this study is to show how the so-called ( $\left.\mathrm{G}^{\prime} / \mathrm{G}\right)$-expansion method [19], which is used for solving nonlinear evolution equations, can be extended to the Brusselator model (1) with the time-space fractional order. The $\left(G^{\prime} / G\right)$-expansion method is an exact solution method which has proven to be a useful tool for constructing exact solutions to FDEs $[20,21]$ as well. The basic idea of the $\left(G^{\prime} / G\right)$-expansion method lies in the fact that it takes full advantage of linear theory via a second order auxiliary equation as the ansatz. With the help of a symbolic computation system such as MATHEMATICA, the $\left(G^{\prime} / G\right)$-expansion method usually provides three types of exact solutions (hyperbolic, trigonometric and rational) with more arbitrary parameters.

The rest of this paper is organized as follows. In Section 2, some preliminaries are given. In Section 3, the method is described. In Section 4, the analytical treatment of our model is presented. In Section 5, some special solutions are construted. Finally, Section 6 is devoted to a concluding remark.

\section{Preliminaries}

In this section, we give a brief background on the definition of the Jumarie's modified Riemann-Liouville derivative. Now, let $f: \mathbb{R} \rightarrow \mathbb{R}, t \rightarrow f(t)$, denote a continuous (but not necessarily differentiable) function. Then the Jumarie's modified Riemann-Liouville derivative of order $\alpha$ is defined as

$$
\begin{gathered}
D_{t}^{\alpha} f(t)=\frac{1}{\Gamma(-\alpha)} \int_{0}^{t}(t-\xi)^{-\alpha-1}(f(\xi)-f(0)) d \xi, \quad \alpha<0, \\
D_{t}^{\alpha} f(t)=\frac{1}{\Gamma(1-\alpha)} \frac{d}{d t} \int_{0}^{t}(t-\xi)^{-\alpha}(f(\xi)-f(0)) d \xi \\
0<\alpha<1, \\
D_{t}^{\alpha} f(t)=\left(f^{(\alpha-n)}(t)\right)^{(n)}, \quad n \leq \alpha<n+1, n \geq 1 .
\end{gathered}
$$

This fractional derivative has been applied to fractional Laplace problems [22], fractional variational calculus [23] and probability calculus [24]. The Jumarie's modified Riemann-Liouville derivative has some interesting properties: (i) the $\alpha$-order derivative of a constant is zero; (ii) it can be applied to both differentiable and nondifferentiable functions. The following formulas are given in [14]:

$$
\begin{gathered}
D_{t}^{\alpha} t^{\gamma}=\frac{\Gamma(1+\gamma)}{\Gamma(1+\gamma-\alpha)} t^{\gamma-\alpha}, \quad \gamma>0, \\
D_{t}^{\alpha}(f(t) g(t))=g(t) D_{t}^{\alpha} f(t)+f(t) D_{t}^{\alpha} g(t), \\
D_{t}^{\alpha} f(g(t))=f_{g}^{\prime}(g(t)) D_{t}^{\alpha} g(t)=D_{g}^{\alpha} f(g(t))\left(g_{t}^{\prime}\right)^{\alpha},
\end{gathered}
$$

These results can be directly derived from the equality $d^{\alpha} x(t)=\Gamma(1+\alpha) d x(t)$.

\section{Methodology}

In a previous work, Li and He [25] showed that FDEs with the Jumarie's modified Riemann-Liouville derivative can be easily turned into ODEs. To this end, they proposed a fractional complex transform which reads

$$
\xi=\frac{p x^{\beta}}{\Gamma(1+\beta)}+\frac{q t^{\alpha}}{\Gamma(1+\alpha)}, \quad 0<\alpha \leq 1,0<\beta \leq 1,
$$

where $p$ and $q$ are constants to be determined. Keeping this fact in mind, let us proceed to recall the basic idea of the $\left(\mathrm{G}^{\prime} / \mathrm{G}\right)$-expansion method. Consider a general fractional differential equation for $u=u(x, t)$ in the form

$P\left(u, D_{t}^{\alpha} u, D_{x}^{\beta} u, D_{t}^{2 \alpha} u, D_{x}^{2 \beta} u, \ldots\right)=0, \quad 0<\alpha \leq 1,0<\beta \leq 1$,

where $P$ is a polynomial of its arguments, while $D_{t}^{r \alpha}$ and $D_{x}^{r \beta}$ denote the collection of Jumarie's modified RiemannLiouville derivative terms (in time) of order $r \alpha$ and (in space) of order $r \beta$, respectively. Then, by means of the fractional complex transform (9), Eq. (10) turns into an ordinary differential equation of the form

$$
P\left(U, q U^{\prime}, p U^{\prime}, q^{2} U^{\prime \prime}, p^{2} U^{\prime \prime}, \ldots\right)=0,
$$

where $u(x, t)=U=U(\xi)$ and the primes denote ordinary derivatives with respect to $\xi$. We assume that the solution(s) of Eq. (11) can be expressed in the form

$$
U=a_{n}\left(\frac{G^{\prime}}{G}\right)^{n}+\cdots
$$

where $G=G(\xi)$ is the solution of the auxiliary linear second order ordinary differential equation

$$
G^{\prime \prime}+\lambda G^{\prime}+\mu G=0,
$$

in which $G^{\prime}=d G / d \xi, G^{\prime \prime}=d^{2} G / d \xi^{2}$, while $a_{1}, a_{0}, \ldots, a_{n}$ $(\neq 0), \lambda$ and $\mu$ are constants to be determined at the stage of solving the problem. The unwritten part in (12) is also a 
polynomial in $\left(\mathrm{G}^{\prime} / \mathrm{G}\right)$, but the degree of which is generally equal to or less than $n-1$. The positive integer $n$ can be identified by the homogeneous balance method to the highest order derivatives and nonlinear terms appearing in Eq. (11). Then the substitution of (12) together with Eq. (13) into Eq. (11) yields a system of nonlinear algebraic equations for $a_{i}$ 's, $\lambda, \mu, p$ and $q$. Let us assume that these constants can be determined by solving the resultant simultaneous algebraic equations with the aid of a symbolic computation system such as MATHEMATICA. On the other hand, from the linear theory, the general solutions of Eq. (13) are well known to us. As a result, we obtain exact solutions for Eq. (10) provided they exist.

(Remark. In a previous work, Aslan [26] proved that one can set the constant $\lambda$ to zero without loss of generality. This approach is more advantageous than the original assumption because the number of the parameters is minimized at the outset. Moreover, it provides equivalent results. To take advantage of this observation, we set $\lambda=0$ in our computations.)

\section{Analysis of Eq. (1)}

To obtain exact solutions for Eq. (1), let us first make the fractional complex transform

$$
\begin{gathered}
u(x, t)=U(\xi), \quad v(x, t)=V(\xi), \\
\xi=\frac{k x^{\beta}}{\Gamma(1+\beta)}-\frac{w t^{\alpha}}{\Gamma(1+\alpha)}+\chi,
\end{gathered}
$$

where $k$ and $w$ are real parameters to be specified, while $\chi$ denotes the phase shift. On substituting (14) into Eq. (1), one gets

$$
\begin{aligned}
& k^{2} K U^{\prime \prime}+w U^{\prime}+U^{2} V-B U=0, \\
& k^{2} K V^{\prime \prime}+w V^{\prime}-U^{2} V+B U=0,
\end{aligned}
$$

where $U=U(\xi), V=V(\xi)$, and the primes denote derivatives with respect to $\xi$. Then our procedure suggests a solution for Eq. (15) in the form

$$
\begin{array}{ll}
U=a_{0}+a_{1}\left(\frac{G^{\prime}}{G}\right), & a_{1} \neq 0, \\
V=b_{0}+b_{1}\left(\frac{G^{\prime}}{G}\right), & b_{1} \neq 0,
\end{array}
$$

where $G=G(\xi)$ satisfies Eq. (13), while the arbitrary constants $a_{0}, a_{1}, b_{0}$ and $b_{1}$ will be determined later. Substituting Eq. (16) together with Eq. (13) into Eq. (15), setting the coefficients of $\left(G^{\prime} / G\right)^{i}(i=0,1,2,3)$ to zero, we derive a system of nonlinear algebraic equations for $a_{0}, a_{1}, b_{0}, b_{1}, \lambda$, $\mu, k$ and $w$. Solving the resulting system simultaneously, we get the relations

$$
\begin{aligned}
& \lambda=0, \quad w=-k \sqrt{2 K\left(B-2 k^{2} K \mu\right)}, \quad a_{0}=\mp \sqrt{B-2 k^{2} K \mu}, \\
& a_{1}=\mp k \sqrt{2 K}, \quad b_{0}=\mp \sqrt{B-2 k^{2} K \mu}, \quad b_{1}= \pm k \sqrt{2 K} \text {, } \\
& \lambda=0, \quad w=k \sqrt{2 K\left(B-2 k^{2} K \mu\right)}, \quad a_{0}=\mp \sqrt{B-2 k^{2} K \mu}, \\
& a_{1}= \pm k \sqrt{2 K}, \quad b_{0}=\mp \sqrt{B-2 k^{2} K \mu}, \quad b_{1}=\mp k \sqrt{2 K} \text {, } \\
& \lambda=0, \quad w=-\frac{B+4 k^{2} K \mu}{2 \sqrt{-\mu}}, \quad a_{0}= \pm k \sqrt{-2 K \mu}, \\
& a_{1}=\mp k \sqrt{2 K}, \quad b_{0}= \pm \frac{B-4 k^{2} K \mu}{2 k \sqrt{-2 K \mu}}, \quad b_{1}= \pm k \sqrt{2 K}, \\
& \lambda=0, \quad w=\frac{B+4 k^{2} K \mu}{2 \sqrt{-\mu}}, \quad a_{0}= \pm k \sqrt{-2 K \mu}, \\
& a_{1}= \pm k \sqrt{2 K}, \quad b_{0}= \pm \frac{B-4 k^{2} K \mu}{2 k \sqrt{-2 K \mu}}, \quad b_{1}=\mp k \sqrt{2 K} .
\end{aligned}
$$

As a result, the relations (17)-(20) lead to the following solutions for Eq. (1):

\subsection{Hyperbolic function solutions}

$$
\begin{aligned}
u(x, t)= & \mp \sqrt{B-2 k^{2} K \mu} \mp k \sqrt{-2 K \mu} \\
& \times\left(\frac{C_{1} \cosh (\sqrt{-\mu} \xi)+C_{2} \sinh (\sqrt{-\mu} \xi)}{C_{1} \sinh (\sqrt{-\mu} \xi)+C_{2} \cosh (\sqrt{-\mu} \xi)},\right. \\
v(x, t)= & \mp \sqrt{B-2 k^{2} K \mu} \pm k \sqrt{-2 K \mu} \\
& \times\left(\frac{C_{1} \cosh (\sqrt{-\mu} \xi)+C_{2} \sinh (\sqrt{-\mu} \xi)}{C_{1} \sinh (\sqrt{-\mu} \xi)+C_{2} \cosh (\sqrt{-\mu} \xi)}\right),
\end{aligned}
$$

where

$$
\xi=\frac{k}{\Gamma(1+\beta)} \chi^{\beta}+\frac{k \sqrt{2 K\left(B-2 k^{2} K \mu\right)}}{\Gamma(1+\alpha)} t^{\alpha}+\chi,
$$

while $k, \chi, \mu(<0), C_{1}$ and $C_{2}$ remain arbitrary (here and henceforth, the signs $( \pm)$ and $(\mp)$ are ordered vertically);

$$
\begin{aligned}
u(x, t)= & \mp \sqrt{B-2 k^{2} K \mu} \pm k \sqrt{-2 K \mu} \\
& \times\left(\frac{C_{1} \cosh (\sqrt{-\mu} \xi)+C_{2} \sinh (\sqrt{-\mu} \xi)}{C_{1} \sinh (\sqrt{-\mu} \xi)+C_{2} \cosh (\sqrt{-\mu} \xi)}\right),
\end{aligned}
$$




$$
\begin{aligned}
v(x, t)= & \mp \sqrt{B-2 k^{2} K \mu} \mp k \sqrt{-2 K \mu} \\
& \times\left(\frac{C_{1} \cosh (\sqrt{-\mu} \xi)+C_{2} \sinh (\sqrt{-\mu} \xi)}{C_{1} \sinh (\sqrt{-\mu} \xi)+C_{2} \cosh (\sqrt{-\mu} \xi)},\right.
\end{aligned}
$$

where

$$
\xi=\frac{k}{\Gamma(1+\beta)} \chi^{\beta}-\frac{k \sqrt{2 K\left(B-2 k^{2} K \mu\right)}}{\Gamma(1+\alpha)} t^{\alpha}+\chi,
$$

while $k, \chi, \mu(<0), C_{1}$ and $C_{2}$ remain arbitrary;

$$
\begin{aligned}
& u(x, t)= \pm k \sqrt{-2 K \mu} \mp k \sqrt{-2 K \mu} \\
& \times\left(\frac{C_{1} \cosh (\sqrt{-\mu} \xi)+C_{2} \sinh (\sqrt{-\mu} \xi)}{C_{1} \sinh (\sqrt{-\mu} \xi)+C_{2} \cosh (\sqrt{-\mu} \xi)}\right), \\
& v(x, t)= \pm \frac{B-4 k^{2} K \mu}{2 k \sqrt{-2 K \mu}} \pm k \sqrt{-2 K \mu} \\
& \times\left(\frac{C_{1} \cosh (\sqrt{-\mu} \xi)+C_{2} \sinh (\sqrt{-\mu} \xi)}{C_{1} \sinh (\sqrt{-\mu} \xi)+C_{2} \cosh (\sqrt{-\mu} \xi)}\right),
\end{aligned}
$$

where

$$
\xi=\frac{k}{\Gamma(1+\beta)} \chi^{\beta}+\frac{B+4 k^{2} K \mu}{2 \sqrt{-\mu} \Gamma(1+\alpha)} t^{\alpha}+\chi,
$$

while $k, \chi, \mu(<0), C_{1}$ and $C_{2}$ remain arbitrary;

$$
\begin{aligned}
u(x, t)= & \pm k \sqrt{-2 K \mu} \pm k \sqrt{-2 K \mu} \\
& \times\left(\frac{C_{1} \cosh (\sqrt{-\mu} \xi)+C_{2} \sinh (\sqrt{-\mu} \xi)}{C_{1} \sinh (\sqrt{-\mu} \xi)+C_{2} \cosh (\sqrt{-\mu} \xi)}\right), \\
v(x, t)= & \pm \frac{B-4 k^{2} K \mu}{2 k \sqrt{-2 K \mu} \mp k \sqrt{-2 K \mu}} \\
& \times\left(\frac{C_{1} \cosh (\sqrt{-\mu} \xi)+C_{2} \sinh (\sqrt{-\mu} \xi)}{C_{1} \sinh (\sqrt{-\mu} \xi)+C_{2} \cosh (\sqrt{-\mu} \xi)}\right),
\end{aligned}
$$

where

$$
\xi=\frac{k}{\Gamma(1+\beta)} \chi^{\beta}-\frac{B+4 k^{2} K \mu}{2 \sqrt{-\mu} \Gamma(1+\alpha)} t^{\alpha}+\chi,
$$

while $k, \chi, \mu(<0), C_{1}$ and $C_{2}$ remain arbitrary.

\subsection{Trigonometric function solutions}

$$
\begin{aligned}
u(x, t)= & \mp \sqrt{B-2 k^{2} K \mu} \mp k \sqrt{2 K \mu} \\
& \times\left(\frac{-C_{1} \sin (\sqrt{\mu} \xi)+C_{2} \cos (\sqrt{\mu} \xi)}{C_{1} \cos (\sqrt{\mu} \xi)+C_{2} \sin (\sqrt{\mu} \xi)},\right. \\
v(x, t)= & \mp \sqrt{B-2 k^{2} K \mu} \pm k \sqrt{2 K \mu} \\
& \times\left(\frac{-C_{1} \sin (\sqrt{\mu} \xi)+C_{2} \cos (\sqrt{\mu} \xi)}{C_{1} \cos (\sqrt{\mu} \xi)+C_{2} \sin (\sqrt{\mu} \xi)}\right),
\end{aligned}
$$

where

$$
\xi=\frac{k}{\Gamma(1+\beta)} \chi^{\beta}+\frac{k \sqrt{2 K\left(B-2 k^{2} K \mu\right)}}{\Gamma(1+\alpha)} t^{\alpha}+\chi,
$$

while $k, \chi, \mu(>0), C_{1}$ and $C_{2}$ remain arbitrary;

$$
\begin{aligned}
u(x, t)= & \mp \sqrt{B-2 k^{2} K \mu} \pm k \sqrt{2 K \mu} \\
& \times\left(\frac{-C_{1} \sin (\sqrt{\mu} \xi)+C_{2} \cos (\sqrt{\mu} \xi)}{C_{1} \cos (\sqrt{\mu} \xi)+C_{2} \sin (\sqrt{\mu} \xi)},\right. \\
v(x, t)= & \mp \sqrt{B-2 k^{2} K \mu} \mp k \sqrt{2 K \mu} \\
& \times\left(\frac{-C_{1} \sin (\sqrt{\mu} \xi)+C_{2} \cos (\sqrt{\mu} \xi)}{C_{1} \cos (\sqrt{\mu} \xi)+C_{2} \sin (\sqrt{\mu} \xi)}\right),
\end{aligned}
$$

where

$$
\xi=\frac{k}{\Gamma(1+\beta)} \chi^{\beta}-\frac{k \sqrt{2 K\left(B-2 k^{2} K \mu\right)}}{\Gamma(1+\alpha)} t^{\alpha}+\chi,
$$

while $k, \chi, \mu(>0), C_{1}$ and $C_{2}$ remain arbitrary.

\subsection{Rational function solutions}

$$
\begin{aligned}
& u(x, t)=\mp \sqrt{B} \mp \frac{k \sqrt{2 K} C_{1}}{C_{1}\left(\frac{k}{\Gamma(1+\beta)} x^{\beta}+\frac{k \sqrt{2 B K}}{\Gamma(1+\alpha)} t^{\alpha}+\chi\right)+C_{2}}, \\
& v(x, t)=\mp \sqrt{B} \pm \frac{k \sqrt{2 K} C_{1}}{C_{1}\left(\frac{k}{\Gamma(1+\beta)} x^{\beta}+\frac{k \sqrt{2 B K}}{\Gamma(1+\alpha)} t^{\alpha}+\chi\right)+C_{2}},
\end{aligned}
$$


where $k, \chi, C_{1}$ and $C_{2}$ remain arbitrary;

$$
\begin{aligned}
& u(x, t)=\mp \sqrt{B} \pm \frac{k \sqrt{2 K} C_{1}}{C_{1}\left(\frac{k}{\Gamma(1+\beta)} x^{\beta}-\frac{k \sqrt{2 B K}}{\Gamma(1+\alpha)} t^{\alpha}+\chi\right)+C_{2}}, \\
& v(x, t)=\mp \sqrt{B} \mp \frac{k \sqrt{2 K} C_{1}}{C_{1}\left(\frac{k}{\Gamma(1+\beta)} x^{\beta}-\frac{k \sqrt{2 B K}}{\Gamma(1+\alpha)} t^{\alpha}+\chi\right)+C_{2}},
\end{aligned}
$$

where $k, \chi, C_{1}$ and $C_{2}$ remain arbitrary.

\section{Further discussion}

The expressions (21)-(36) provide abundant exact solutions for Eq. (1) containing more arbitrary parameters. If desired, one can construct some special solutions of physical interest.

For a first example, if we set " $C_{1} \neq 0$ and $C_{2}=0$ " or " $C_{1}=0$ and $C_{2} \neq 0$ " in Eqs. (21) and (22), respectively, then we get formal topological solitons for Eq. (1) which read

$$
\begin{aligned}
& u(x, t)=\mp \sqrt{B-2 k^{2} K \mu} \mp k \sqrt{-2 K \mu} \operatorname{coth}\left(\sqrt{-\mu}\left(\frac{k}{\Gamma(1+\beta)} x^{\beta}+\frac{k \sqrt{2 K\left(B-2 k^{2} K \mu\right)}}{\Gamma(1+\alpha)} t^{\alpha}+\chi\right),\right. \\
& v(x, t)=\mp \sqrt{B-2 k^{2} K \mu} \pm k \sqrt{-2 K \mu} \operatorname{coth}\left(\sqrt{-\mu}\left(\frac{k}{\Gamma(1+\beta)} x^{\beta}+\frac{k \sqrt{2 K\left(B-2 k^{2} K \mu\right)}}{\Gamma(1+\alpha)} t^{\alpha}+\chi\right),\right.
\end{aligned}
$$

where $k, \chi$ and $\mu(<0)$ remain arbitrary;

$$
\begin{aligned}
& u(x, t)=\mp \sqrt{B-2 k^{2} K \mu} \mp k \sqrt{-2 K \mu} \tanh \left(\sqrt{-\mu}\left(\frac{k}{\Gamma(1+\beta)} x^{\beta}+\frac{k \sqrt{2 K\left(B-2 k^{2} K \mu\right)}}{\Gamma(1+\alpha)} t^{\alpha}+\chi\right),\right. \\
& v(x, t)=\mp \sqrt{B-2 k^{2} K \mu} \pm k \sqrt{-2 K \mu} \tanh \left(\sqrt{-\mu}\left(\frac{k}{\Gamma(1+\beta)} x^{\beta}+\frac{k \sqrt{2 K\left(B-2 k^{2} K \mu\right)}}{\Gamma(1+\alpha)} t^{\alpha}+\chi\right),\right.
\end{aligned}
$$

where $k, \chi$ and $\mu(<0)$ remain arbitrary.

For a second example, if we set " $C_{1} \neq 0$ and $C_{2}=0$ " then we get singular periodic solutions for Eq. (1) which or " $C_{1}=0$ and $C_{2} \neq 0$ " in Eqs. (29) and (30), respectively, read

$$
\begin{aligned}
& u(x, t)=\mp \sqrt{B-2 k^{2} K \mu} \pm k \sqrt{2 K \mu} \tan \left(\sqrt{\mu}\left(\frac{k}{\Gamma(1+\beta)} \chi^{\beta}+\frac{k \sqrt{2 K\left(B-2 k^{2} K \mu\right)}}{\Gamma(1+\alpha)} t^{\alpha}+\chi\right),\right. \\
& v(x, t)=\mp \sqrt{B-2 k^{2} K \mu} \mp k \sqrt{2 K \mu} \tan \left(\sqrt{\mu}\left(\frac{k}{\Gamma(1+\beta)} \chi^{\beta}+\frac{k \sqrt{2 K\left(B-2 k^{2} K \mu\right)}}{\Gamma(1+\alpha)} t^{\alpha}+\chi\right),\right.
\end{aligned}
$$

where $k, \chi$ and $\mu(>0)$ remain arbitrary;

$$
\begin{aligned}
& u(x, t)=\mp \sqrt{B-2 k^{2} K \mu} \mp k \sqrt{2 K \mu} \cot \left(\sqrt{\mu}\left(\frac{k}{\Gamma(1+\beta)} x^{\beta}+\frac{k \sqrt{2 K\left(B-2 k^{2} K \mu\right)}}{\Gamma(1+\alpha)} t^{\alpha}+\chi\right),\right. \\
& v(x, t)=\mp \sqrt{B-2 k^{2} K \mu} \pm k \sqrt{2 K \mu} \cot \left(\sqrt{\mu}\left(\frac{k}{\Gamma(1+\beta)} x^{\beta}+\frac{k \sqrt{2 K\left(B-2 k^{2} K \mu\right)}}{\Gamma(1+\alpha)} t^{\alpha}+\chi\right),\right.
\end{aligned}
$$

where $k, \chi$ and $\mu(>0)$ remain arbitrary. 
For a third example, if we set $\mu=-\left(B / 4 k^{2} K\right)<0$ in respectively, then we get non-constant steady-state soluEqs. (25) and (26) or $\mu=\left(B / 2 k^{2} K\right)>0$ in Eqs. (29) and (30), tions for Eq. (1) which read

$$
\begin{aligned}
& u(x, t)= \pm \sqrt{\frac{B}{2}} \mp \sqrt{\frac{B}{2}}\left(\frac{C_{1} \cosh \left(\sqrt{\frac{B}{4 k^{2} K}}\left(\frac{k}{\Gamma(1+\beta)} x^{\beta}+\chi\right)\right)+C_{2} \sinh \left(\sqrt{\frac{B}{4 k^{2} K}}\left(\frac{k}{\Gamma(1+\beta)} x^{\beta}+\chi\right)\right)}{C_{1} \sinh \left(\sqrt{\frac{B}{4 k^{2} K}}\left(\frac{k}{\Gamma(1+\beta)} x^{\beta}+\chi\right)\right)+C_{2} \cosh \left(\sqrt{\frac{B}{4 k^{2} K}}\left(\frac{k}{\Gamma(1+\beta)} x^{\beta}+\chi\right)\right)}\right), \\
& v(x, t)= \pm \sqrt{2 B} \pm \sqrt{\frac{B}{2}}\left(\frac{C_{1} \cosh \left(\sqrt{\frac{B}{4 k^{2} K}}\left(\frac{k}{\Gamma(1+\beta)} x^{\beta}+\chi\right)+C_{2} \sinh \left(\sqrt{\frac{B}{4 k^{2} K}}\left(\frac{k}{\Gamma(1+\beta)} x^{\beta}+\chi\right)\right)\right.}{C_{1} \sinh \left(\sqrt{\frac{B}{4 k^{2} K}}\left(\frac{k}{\Gamma(1+\beta)} x^{\beta}+\chi\right)\right)+C_{2} \cosh \left(\sqrt{\frac{B}{4 k^{2} K}}\left(\frac{k}{\Gamma(1+\beta)} x^{\beta}+\chi\right)\right)}\right),
\end{aligned}
$$

where $k, \chi, C_{1}$ and $C_{2}$ remain arbitrary;

$$
\begin{aligned}
& u(x, t)=\mp \sqrt{B}\left(\frac{-C_{1} \sin \left(\sqrt{\frac{B}{2 k^{2} K}}\left(\frac{k}{\Gamma(1+\beta)} x^{\beta}+\chi\right)\right)+C_{2} \cos \left(\sqrt{\frac{B}{2 k^{2} K}}\left(\frac{k}{\Gamma(1+\beta)} x^{\beta}+\chi\right)\right)}{C_{1} \cos \left(\sqrt{\frac{B}{2 k^{2} K}}\left(\frac{k}{\Gamma(1+\beta)} x^{\beta}+\chi\right)\right)+C_{2} \sin \left(\sqrt{\frac{B}{2 k^{2} K}}\left(\frac{k}{\Gamma(1+\beta)} x^{\beta}+\chi\right)\right)},\right. \\
& v(x, t)= \pm \sqrt{B}\left(\frac{-C_{1} \sin \left(\sqrt{\frac{B}{2 k^{2} K}}\left(\frac{k}{\Gamma(1+\beta)} x^{\beta}+\chi\right)\right)+C_{2} \cos \left(\sqrt{\frac{B}{2 k^{2} K}}\left(\frac{k}{\Gamma(1+\beta)} x^{\beta}+\chi\right)\right)}{C_{1} \cos \left(\sqrt{\frac{B}{2 k^{2} K}}\left(\frac{k}{\Gamma(1+\beta)} x^{\beta}+\chi\right)\right)+C_{2} \sin \left(\sqrt{\frac{B}{2 k^{2} K}}\left(\frac{k}{\Gamma(1+\beta)} x^{\beta}+\chi\right)\right)}\right),
\end{aligned}
$$

where $k, \chi, C_{1}$ and $C_{2}$ remain arbitrary.

Like manner, we can extract some other non-constant steady-state solutions for Eq. (1) from the expressions (27) and (28) as well (31) and (32). We skip this procedure for the sake of brevity.

\section{Conclusion}

In recent years, FDEs have been received much attention due to their numerous applications in the areas of physics, biology and engineering. In this paper, a generalization of the Brusselator model to fractional order (in the sense of Jumarie) is proposed. By using the $\left(\mathrm{G}^{\prime} / \mathrm{G}\right)$-expansion method we have successfully obtained three types of exact solutions with arbitrary parameters; hyperbolic, trigonometric and rational. We have also obtained exact solutions in terms of topological solitons and singular periodic functions as well as non-constant steady-states. Our results are subject to some adequate physical interpretations in the future.

Received: June 28, 2011. Accepted: January 6, 2014.

\section{References}

[1] K.B. Oldham, J. Spanier, The Fractional Calculus, Academic Press, New York, London, 1974.

[2] K.S. Miller, B. Ross, An Introduction to the Fractional Calculus and Fractional Differential Equations, Wiley, New York, 1993.

[3] S.G. Samko, A.A. Kilbas, O.I. Marichev, Fractional Integrals and Derivatives. Theory and Applications, Gordon and Breach, Yverdon, 1993.

[4] I. Podlubny, Fractional Differential Equations, Academic Press, San Diego, 1999.

[5] A.A. Kilbas, H.M. Srivastava, J.J. Trujillo, Theory and Applications of Fractional Differential Equations, Elsevier, San Diego, 2006. 
[6] Z. Odibat, S. Momani, The variational iteration method: An efficient scheme for handling fractional partial differential equations in fluid mechanics, Comput. Math. Appl. 58 (2009) 2199-2208.

[7] J.H. He, A coupling method of a homotopy technique and a perturbation technique for non-linear problems, Int. J. Nonlinear Mech. 35 (2000) 37-43.

[8] A.M.A. El-Sayed, M. Gaber, The Adomian decomposition method for solving partial differential equations of fractal order in finite domains, Phys. Lett. A 359 (2006) 175-182.

[9] Z. Odibat, S. Momani, A generalized differential transform method for linear partial differential equations of fractional order, Appl. Math. Lett. 21 (2008) 194-199.

[10] M. Cui, Compact finite difference method for the fractional diffusion equation, J. Comput. Phys. 228 (2009) 7792-7804.

[11] Q. Huang, G. Huang, H. Zhan, A finite element solution for the fractional advection-dispersion equation, Adv. Water Resour. $31(2008)$ 1578-1589.

[12] B. Lu, The first integral method for some time fractional differential equations, J. Math. Anal. Appl. 395 (2012) 684-693.

[13] S. Zhang, H.Q. Zhang, Fractional sub-equation method and its applications to nonlinear fractional PDEs, Phys. Lett. A 375 (2011) 1069-1073.

[14] G. Jumarie, Modified Riemann-Liouville derivative and fractional Taylor series of nondifferentiable functions further results, Comput. Math. Appl. 51 (2006) 1367-1376.

[15] I. Prigogine, R. Lefever, Symmetry breaking instabilities in dissipative systems: II, J. Chem. Phys. 48 (1968) 1665-1700.
[16] R. Lefever, G. Nicolis, Chemical instabilities and sustained oscillations, J. Theor. Biol. 30 (1971) 267-284.

[17] G. Nicolis, I. Prigogine, Self-Organization in Non-Equilibrium Systems, Wiley, New York, 1977.

[18] J. Tyson, Some further studies of non-linear oscillations in chemical systems, J. Chem. Phys. 58 (1973) 3919-3930.

[19] M. Wang, X. Li, J. Zhang, The (G'/G)-expansion method and traveling wave solutions of nonlinear evolution equations in mathematical physics, Phys. Lett. A. 372 (2008) 417-423.

[20] Z. Bin, (G'/G)-expansion method for solving fractional partial differential equations in the theory of mathematical physics, Commun. Theor. Phys. 58 (2012) 623-630.

[21] K.A. Gepreel, S. Omran, Exact solutions for nonlinear partial fractional differential equations, Chin. Phys. B 21 (2012) 110204.

[22] G.C. Wu, E.W.M. Lee, Fractional variational iteration method and its application, Phys. Lett. A 374 (2010) 2506-2509.

[23] G. Jumarie, New stochastic fractional models for Malthusian growth, the Poissonian birth process and optimal management of populations, Math. Comput. Model. 44 (2006) 231-254.

[24] G. Jumarie, Laplace's transform of fractional order via the Mittag-Leffler function and modified Riemann-Liouville derivative, Appl. Math. Lett. 22 (2009) 1659-1664.

[25] Z.B. Li, J.H. He, Fractional complex transformation for fractional differential equations, Math. Comput. Appl. 15 (2010) 970-973.

[26] I. Aslan, A note on the ( $\left.G^{\prime} / G\right)$-expansion method again, Appl. Math. Comput. 217 (2010) 937-938. 